\title{
The Impact of Surface Plasmon Resonance in Virology \\ Marco Rusnati*
}

Unit of General Pathology and Immunology, Department of Biomedical Sciences and Biotechnology, School of Medicine, University of Brescia, Italy

Viruses are the etiological cause of important diseases worldwide, and, despite decades of drug research and development, they are still a top global healthcare problem. These considerations make virus detection, the study of their mechanisms of action and the consequent identification of new antiviral drugs extremely important for medical healthcare.

Although very simple if compared to eukaryotic cells, viruses are characterized by heterogeneous structures and mechanisms of action and are prone to high rate of mutation, making their study very diversified and difficult. At a molecular level, few proteins forming a virus "hijack" numerous host structures both at an extracellular and intracellular level, compete out physiological ligands and take control of a whole eukaryotic cell.

Unfortunately, the systematic study of countless virus/host interactions is quite complex, time-consuming and expensive, calling for new high-throughput methodologies. To this aim, in the last years, proteomic, genomic and computational biology approaches have been exploited in the field of virology providing an incessant torrent of "omics" data functional to the definition of the closely related viral "interactome", "infectome" and "diseasome".

Surface plasmon resonance (SPR) is a handy-user, reliable, and high-throughput optical technique to evaluate biomolecular interactions. Launched less than 20 years ago, its use in virology has seen tremendous growth and this trend is predicted to continue as the technology becomes more accessible and its applications more diverse. Briefly, SPR allows the evaluation of biomolecular interactions by detecting reflected light from a prism-gold film interface. A receptor specific for a particular analyte is chemically immobilized onto the gold film. When the sensor is exposed to a sample containing the analyte, its binding to the receptor can be monitored as a real-time graph of the response units (RU) against time (sensorgram) (Figure 1) [1]. Due to its peculiar architecture, in respect to conventional techniques such as fluorescent, enzyme- or radio-labelled assays, SPR adds to labelfree molecular recognition several advantages including the ability to investigate and manipulate minute concentrations of molecules

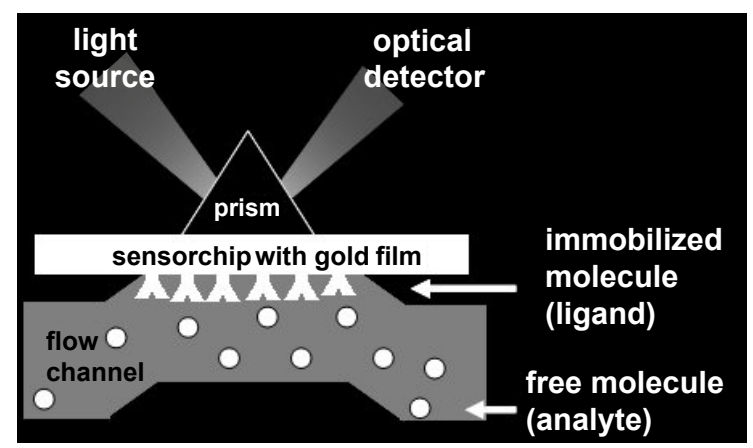

The molecule immobilized onto the gold film is named ligand whereas the analyte is the putative partner injected into the microfluidic system. In the presence of an interaction, the refractive index at the metal surface change, resulting in the shift of the resonance angle and providing label-free transduction of the binding reaction.

Figure 1: Schematic representation of an SPR apparatus. semi-automatically and the possibility to detect analytes over a wide range of molecular weights and binding affinities, spanning from huge intact virions to weakly interacting small antiviral pro-drugs. SPR has been already successfully exploited to study different aspect of virus biology and anti-viral therapy: virus genotyping; detection and quantification of viruses; viral components or anti-virus antibodies in body fluids; mapping of viral epitopes and characterization of antiviral antibodies; screening of library of putative antiviral pro-drugs and lead product characterization. However, the most important and promising developments of SPR in virology can be foreseen in the study of those macromolecular interactions that collectively account for the so called "virus/host interactome". A systematic, standardized and coordinated use of SPR can indeed lead to the classification of these interactions into different categories on the bases of their biological function: 1) interactions of viral envelope proteins (determinant of infectivity) with host cell surface structures (entry receptors) during the early phases of infection; 2) interactions of internalized virus (or its components) with intracellular host proteins or 3) with nucleic acids, required for virus replication and for the assembly of new virions; 4) interactions of newly synthesized viral components with proteins of the cellular secretory pathway for virus spread; 5) interactions of "virokines" or "viroceptors" (viral protein released by infected cells) with surrounding uninfected cells, functional to the creation of an environment favourable to the further dissemination of the virus. Relevantly, each of these interactions can be taken in account as a therapeutical target for the development of new antiviral drugs.

Also, by a systematic, standardized and coordinated use of SPR, each virus/host interactions (or each category outlined above) can be ranked on the basis of their affinities, identifying those occurring with the lowest strength (hopefully corresponding to the highest drug ability).

Finally, our experience with SPR validated its use for the identification of host structures that act as "nodal points" in the biology of a given virus. As an example, we found that heparan sulfate proteoglycans interact with at least three different proteins of the human immunodeficiency virus (HIV), being thus deeply implicated in different phases of the virus cycle, and emerging as promising target for multitargeted antiviral therapies [2].

*Corresponding author: Marco Rusnati, Unit of General Pathology \& Immunology, Department of Biomedical Sciences and Biotechnology, viale Europa 11, 25123 Brescia, Italy, Tel.: +39-0303717315; Fax: +39-0303701157; E-mail: rusnati@med.unibs.it

Received October 19, 2012; Accepted October 22, 2012; Published October 25, 2012

Citation: Rusnati M (2012) The Impact of Surface Plasmon Resonance in Virology. J Bioengineer \& Biomedical Sci 2:e108. doi:10.4172/2155-9538.1000e108

Copyright: (c) 2012 Rusnati M. This is an open-access article distributed under the terms of the Creative Commons Attribution License, which permits unrestricted use, distribution, and reproduction in any medium, provided the original author and source are credited. 
Citation: Rusnati M (2012) The Impact of Surface Plasmon Resonance in Virology. J Bioengineer \& Biomedical Sci 2:e108. doi:10.4172/21559538.1000 e108

Page 2 of 2

In conclusion, SPR may ideally integrate computational and experimental chemical systems biology approaches starting from the finely detailed characterization of relevant macromolecular interactions, that will help in drawing "connectivity maps" providing better tools for drug discovery in respect to traditional 'monotargetcentric' drug screening. New drugs developed in these contexts may function as global inhibitors, simultaneously disabling multiple networks of virus biology.

\section{Acknowledgement}

This work was supported by grants from MIUR, ISS (AIDS Project) to Marco Rusnati.

\section{References}

1. Rich RL, Myszka DG (2000) Survey of the 1999 surface plasmon resonance biosensor literature. J Mol Recognit 13: 388-407.

2. Rusnati M, Vicenzi E, Donalisio M, Oreste P, Landolfo S, et al. (2009) Sulfated K5 Escherichia coli polysaccharide derivatives: A novel class of candidate antiviral microbicides. Pharmacol Ther 123: 310-322. 